\title{
A study of bacterial growth on the skin surface after a basketball game
}

\author{
Nobuhiko Eda ${ }^{*}$, Hironaga Ito ${ }^{2}$, Kazuhiro Shimizu ${ }^{3}$, Satomi Suzuki ${ }^{4}$, Eunjae Lee ${ }^{4}$ and Takao Akama \\ ${ }^{1}$ Faculty of Sport Sciences, Waseda University, Saitama, Japan \\ ${ }^{2}$ Graduate School of Sport Sciences, Waseda University, Saitama, Japan \\ ${ }^{3}$ Department of Sports Science, Japan Institute of Sports Sciences, Tokyo, Japan \\ ${ }^{4}$ Waseda Institute for Sport Sciences, Waseda University, Saitama, Japan
}

\begin{abstract}
The aim of this study was to examine the change in number of staphylococci on a player's skin after a basketball game, using the nutrient agar method. Six healthy males (age, $21.8 \pm 2.6$ years) participated in this study. Measurements were carried out before and after a basketball game. Staphylococci were detected by pressing agar-based media against the skin surface of the middle chest and the medial side of the forearm of each study participant. After incubation, the black colony surrounding media got clouded (Staphylococcus aureus; S. aureus) and other black colony (coagulase-negative staphylococcus; CNS) were counted using the colony forming unit (CFU). The number of CNS on the chest and forearm significantly increased after the game (chest before game $66.5 \pm 90.2 \mathrm{CFU}$; chest after game $199.5 \pm 155.5 \mathrm{CFU} ; \mathrm{p}<0.05$; forearm before game, $5.3 \pm 8.8 \mathrm{CFU}$; forearm after game $84.7 \pm 77.7 \mathrm{CFU}$; $\mathrm{p}<0.05)$. No colony forming units of S. aureus were identified on the chest and the forearm of players before the game. However, colony forming units of S. aureus were identified on two players' chests (4 and $6 \mathrm{CFU}$ ) and one player's forearm (7 CFU) after the game. Our findings suggest that playing contact sports lead to a higher risk of staphylococcal skin infections than playing non-contact sports. From a practical point of view, we recommend that athletes shower immediately refrain from vigorous scrubbing of the skin after participating in sports activities.
\end{abstract}

\section{Introduction}

Various skin infections, such as tinea and herpes simplex and staphylococcal infections, are particularly common among athletes [1]. Athletes' skin often provides a fertile breeding environment for microorganisms because the surface is often macerated from sweating [2]. A previous study in our laboratory reported that high intensity endurance exercise may compromise the physical barrier of the skin surface due to swelling of the stratum corneum and the secretion of natural moisturizing factor (NMF) during sweating [3]. Additionally, scrapes and scratches caused by sporting activities can damage the cutaneous barrier of the skin thereby allowing microorganisms to infiltrate deeper layers [2]. Therefore, it is important for athletes to keep their skin surface in good condition to prevent skin infections.

In particular, staphylococcal skin infections such as impetigo, cellulitis, abscess, furuncles, and carbuncles often occur in players participating in contact sports [1,4]. Staphylococcus aureus (S. aureus) and coagulase negative staphylococci (CNS) are among the most common staphylococci resident on the skin surface. S. aureus is the most common bacteria causing staphylococcal skin infections. Fontanilla et al. [5] reported that 67 football players and athlete trainers were positive for $S$. aureus, of which $8(12 \%)$ developed staphylococcal skin infections. Skin contact with other athletes and with contaminated sports equipment is thought to contribute to increases in $S$. aureus infections in athletes [6]. Staphylococcus epidermidis (S. epidermidis) is the most frequently isolated CNS species on the skin and it expresses a virulence factor that allows it to attach more tightly to the surface of epithelial cells than strains of S. aureus [7]. S. epidermidis also forms biofilms [8]. In immunocompromised hosts and patients with a damaged cutaneous barrier, S. epidermidis can cause skin infections, such as folliculitis and sycosis vulgaris [9]. Therefore, athletes may be at increased risk for skin infections due to immunosuppression induced by intensive exercise [1], and countermeasures against infectious disease of the skin could play a significant role in maintaining performance conditioning in athletes.

To ensure that athletes remain in playing condition, it is important that methods easy and non-invasive methods are used to measure skin condition. We have used a simple nutritional agar to determine the numbers of bacteria present on the skin surface of athletes, and we have investigated the effects of high intensity peddling exercise on epidermal barriers and skin condition in athletes $[3,10]$. The goal of the current study was to examine the change in the number of staphylococci present on player's skin surface before and after engaging in a contact sport, using the nutrient agar method as an assay.

\section{Materials and methods}

\section{Participants}

Six healthy males (age, $21.8 \pm 2.6$ years) participated in the study. The participants belonged to a basketball team and participated in a

Correspondence to: Nobuhiko Eda, Faculty of Sport Sciences, Waseda University, 2-579-15 Mikajima, Tokorozawa, Saitama 359-1192, Japan, Tel: 81-4-2947-6721; Fax: 81-4-2947-6721; E-mail: nob-eda@aoni.waseda.jp

Key words: Staphylococcus, skin infection, contact sport, athlete

Received: November 11, 2015; Accepted: December 02, 2015; Published: December 05, 2015 
basketball game. Measurements of bacterial growth were carried out before and after the game. All participants in the study received a detailed explanation of the risks, adverse effects, and potential benefits of the study. Informed consent was obtained from all of the participants and all participants had passed a complete medical examination within the preceding year. No participants had been treated with any drugs that were known to affect immune function, and none reported allergies or recent infections within the prior 3 months. Experiments were conducted in accordance with the Declaration of Helsinki.

\section{Staphylococci}

S. aureus and CNS were detected by pressing agar-based media composed of tellurite, glycine, salt, and egg yolk (Food Stamp; Nissui Pharmaceutical, Tokyo, Japan) against the skin surface of the middle chest and the medial side of the forearm of each study participant according to the procedures established in previous studies $[3,10]$. Samples were cultured in a $\mathrm{CO}_{2}$ incubator (B111-9; Sanyo Electric, Osaka, Japan) at $37^{\circ} \mathrm{C}$ for three days, after which black colonies surrounding the media got clouded (S. aureus) and the other black colony (CNS) were counted using the colony forming unit (CFU) (Figure 1).

\section{Statistical analyses}

Descriptive data are presented as mean \pm SD. A paired t-test was used to assess the differences in bacterial populations before and after the basketball game. For all analyses, $\mathrm{p}<0.05$ was set as the threshold for forearm statistical significance.

\section{Results}

The number of CNS on the chest before the game was $66.5 \pm 90.2$ CFU and that after the game was $199.5 \pm 155.5 \mathrm{CFU}$ (Figure 2a). In
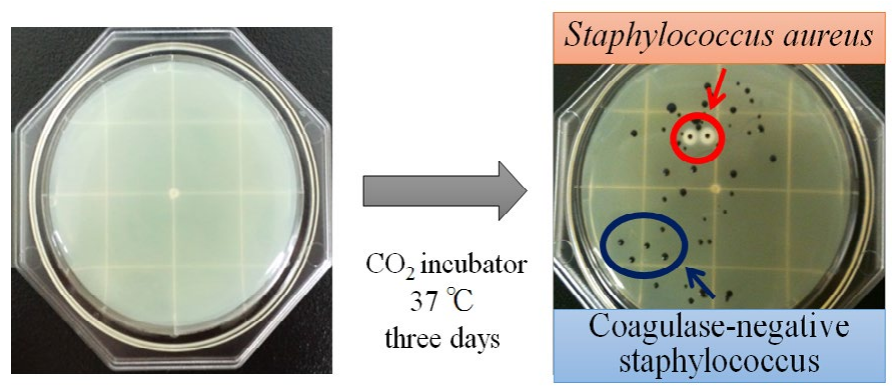

Figure 1. Samples were cultured in a $\mathrm{CO}_{2}$ incubator at $37^{\circ} \mathrm{C}$ for three days, after which black colonies surrounding the media got clouded (Staphylococcus aureus; S. aureus) and the other black colony (coagulase-negative staphylococcus; CNS) were counted. (a)

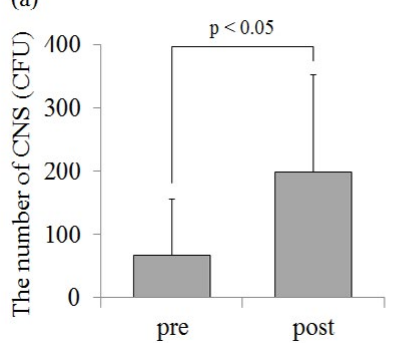

(b)

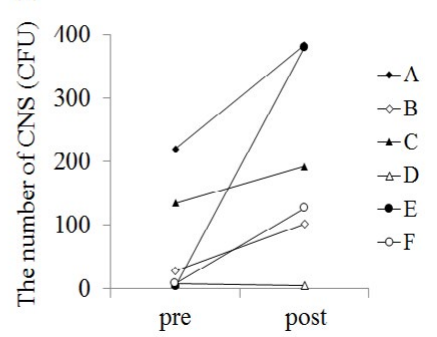

Figure 2. (a) - The average value of the number of coagulase-negative staphylococcus (CNS) on the chest before (pre) and after (post) the game. (b) - The individual data of the number of CNS on the chest before and after the game.
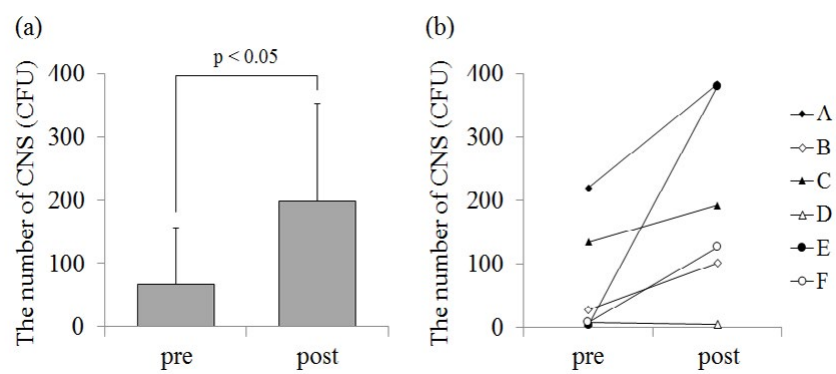

Figure 3. (a) - The average value of the number of coagulase-negative staphylococcus (CNS) on the forearm before (pre) and after (post) the game. (b) - The individual data of the number of CNS on the forearm before and after the game.

Table 1. The individual data of the number of staphylococcus aureus before (pre) and after (post) the game.

\begin{tabular}{|c|c|c|c|c|}
\hline & \multicolumn{2}{|c|}{ chest } & \multicolumn{2}{c|}{ forearm } \\
\hline & pre & post & pre & post \\
\hline Player A & 0 & 0 & 0 & 0 \\
\hline B & 0 & 0 & 0 & 0 \\
\hline C & 0 & 6 & 0 & 0 \\
\hline D & 0 & 0 & 0 & 0 \\
\hline E & 0 & 4 & 0 & 7 \\
\hline F & 0 & 0 & 0 & 0 \\
\hline
\end{tabular}

Colony forming unit (CFU)

five players (player A, B, C, E, F), CNS on the chest increased after the game $(\mathrm{p}<0.05$; Figure $2 \mathrm{~b})$. CNS on the skin surface of player $\mathrm{D}$ was very small in number and there was little change before and after the game. The number of CNS on the forearm before the game was $5.3 \pm 8.8 \mathrm{CFU}$ and that after the game was $84.7 \pm 77.7 \mathrm{CFU}$ (Figure $3 \mathrm{a}$ ). In five players (player A, B, C, E, F), CNS on the forearm also increased after the game. The number of CNS on the chest $(p<0.05$; Figure 2$)$ and the forearm $(\mathrm{p}<0.05$; Figure 3$)$ significantly increased after the game.

No colony forming units of $S$. aureus were identified on the chest and the forearm of players before the game (Table 1). However, colony forming units of $S$. aureus were identified on two players' chests ( 4 and $6 \mathrm{CFU}$ ) and one player's forearm (7 CFU) after the game (Table 1).

\section{Discussion}

The aim of this study was to examine the change in number of staphylococci on a player's skin after a basketball game. We could measure the change in staphylococci on the skin surface using the nutrient agar method established in our previous studies $[3,10]$. In the current study, we demonstrated that the number of CNS present on the player's skin increased, and that $S$. aureus was present on player's skin after the game.

The number of CNS on the chest and forearm significantly increased after the game. Our previous study reported that the number of staphylococci, defined as the total number of $S$. aureus and CNS, increased after non-contact exercise such as a high intensity peddling exercise $[3,10]$. In the present study, involving a basketball game as contact sport, we also demonstrated that the number of CNS increased after the game. S. epidermidis, which is the most frequently isolated species of CNS, has a beneficial role in the skin microflora by producing its own antimicrobial peptides [11] and enhancing the 
expression of human antimicrobial peptides from keratinocytes [12]. On the other hand, S. epidermidis also expresses a virulence factor that allows it to attach more tightly to the surface of epithelial cells when compared to other staphylococcal species [7] and it form biofilms [8]. Skin infections, such as folliculitis and sycosis vulgaris, can be caused by $S$. epidermidis in immunocompromised hosts and patients with a damaged cutaneous barrier [9]. Therefore, an increase in CNS after contact sports can promote infectious skin disease especially in athletes with immunosuppression induced by intensive exercise or skin wounds [1].

No colony forming units of $S$. aureus were identified on the chest and the forearm of players before the game. However, colony forming units of $S$. aureus were identified on two players' chests and one player's forearm after the game. Previous studies have demonstrated that skin infection caused by $S$. aureus is a serious problem for athletes [13-15], and these infections may have an adverse effect on athlete conditioning and performance $[1,5,16]$. Sosin et al. [15] investigated an outbreak of furuncles in 124 male high school athletes. The overall attack rate reported in the study was $25 \%(31 / 124)$ and the risk of developing furuncles two to three times higher in high school athletes with skin injuries. In addition, it is known that increases in skin colonization of $S$. aureus correspond to increased moisture content of the stratum corneum, and that skin exposed to excess water is more susceptible to bacterial infection [17]. Therefore, we hypothesized that skin wounds and sweating during a basketball game might increase a player's risk of skin infections.

Staphylococci are resident bacteria in the normal microflora of the skin. A previous study reported that skin wounds may promote the appearance of staphylococci from sebaceous glands [18]. Additionally, the doubling time for staphylococci is $28.8 \mathrm{~min}$ at $37^{\circ} \mathrm{C} \mathrm{[19].} \mathrm{Therefore,}$ it is possible that staphylococci exit through the pores with sweat and propagate due to an increase in skin temperature during exercise. Our previous studies reported that few colony forming units of $S$. aureus were measured on the chest and the forearm both before and after non-contact exercise $[3,10]$. However, in the present study, S. aureus appeared on the skin surface after the contact exercise during the basketball game. Players of contact sports often have skin contact with other players and activities can result in skin wounds. Therefore, it is possible that, due to increased skin contact and the occurrence of skin wounds, players of contact sports may have a higher risk of contracting staphylococcal skin infections than players of non-contact sports.

In the present study, we could measure colony forming units of CNS and S. aureus on basketball players' skin easily and non-invasively, using a nutrient agar. We demonstrated that numbers of CNS and $S$. aureus increased on players' skin after the basketball game, and this phenomenon could lead to an increased risk of staphylococcal skin infections in these players. Skin infections may negatively affect athlete performance; therefore, countermeasures against infectious diseases of the skin are necessary for maintaining playing condition in athletes. From a practical point of view, we encourage athletes to take a shower immediately after any sports activities. However, Yamada et al. [18] suggested that cleaning skin with a brush may scratch the skin surface and promote the appearance of staphylococci from sebaceous glands. Therefore, it may important for athletes to shower without vigorous scrubbing in order to prevent skin infections $[18,20]$. Future studies are required for further examination of the time course of appearance of colony forming units of CNS and S. aureus on the skin surface after a basketball game, and further investigation of showering practices intended to prevent staphylococcal skin infections.

\section{Conclusion}

We could measure the change in colony forming units of CNS and S. aureus on the skin surface after a basketball game easily and noninvasively, using a nutrient agar. Consequently, we revealed that the number of colonies of CNS increased, and S. aureus newly appeared, on the skin after the game. Our findings suggest that playing contact sports lead to a higher risk of staphylococcal skin infections than playing noncontact sports. From a practical point of view, we recommend that athletes shower immediately refrain from vigorous scrubbing of the skin after participating in sports activities.

\section{Conflict of interest}

We have no financial interest or no conflict of interest.

\section{Acknowledgements}

We thank all of the subjects for participating in this study. This study was supported by the Grant-in-Aid for the Global COE "Sport Sciences for the Promotion of Active Life" from the Ministry of Education, Culture, Sports, Science and Technology of Japan.

\section{References}

1. Pecci M, Comeau D, Chawla V (2009) Skin conditions in the athlete. Am J Sports Med 37: 406-418. [Crossref]

2. Adams BB (2008) Skin infections in athletes. Dermatol Nurs 20: 39-44. [Crossref]

3. Eda N, Shimizu K, Suzuki S, Lee E, Akama T (2013) Effects of High-Intensity Endurance Exercise on Epidermal Barriers against Microbial Invasion. J Sports Sci Med 12: 44-51. [Crossref]

4. Adams BB (2002) Dermatologic disorders of the athlete. Sports Med 32: 309-321. [Crossref]

5. Fontanilla JM, Kirkland KB, Talbot EA, Powell KE, Schwartzman JD, et al. (2010) Outbreak of skin infections in college football team members due to an unusual strain of community-acquired methicillin-susceptible Staphylococcus aureus. J Clin Microbiol 48: 609-611. [Crossref]

6. Pollard JG (1967) The staphylococcus plagues a football team. J Am Coll Health Assoc 15: 234-238.

7. Kume M, Fukushima H (1994) Adherence to epithelial cells and enzyme production of methicillin-resistant, coagulase-negative staphylococci. Journal of Osaka Odontological Society 57: 1-16.

8. Raad I, Alrahwan A, Rolston K (1998) Staphylococcus epidermidis: emerging resistance and need for alternative agents. Clin Infect Dis 26: 1182-1187. [Crossref]

9. Katayama I, Tsuchida T, Hashimoto T, Furue M, Watanabe S (2009) Dermatology (3rdedn), Bunkodo CO., LTD, Tokyo.

10. Eda N Shimizu K, Suzuki S, Tanabe Y, Lee E, et al. (2013) Altered secretory immunoglobulin A on skin surface after intensive exercise. J Strength Cond Res 27: 2581-2587. [Crossref]

11. Cogen AL, Yamasaki K, Sanchez KM, Dorschner RA, Lai Y, et al. (2010) Selective antimicrobial action is provided by phenol-soluble modulins derived from Staphylococcus epidermidis, a normal resident of the skin. J Invest Dermatol 130: 192200. [Crossref]

12. Dinulos JG, Mentele L, Fredericks LP, Dale BA, Darmstadt GL (2003) Keratinocyte expression of human beta defensin 2 following bacterial infection: role in cutaneous host defense. Clin Diagn Lab Immunol 10: 161-166. [Crossref]

13. Bartlett PC, Martin RJ, Cahill BR (1982) Furunculosis in a high school football team Am J Sports Med 10: 371-374. [Crossref]

14. Lindenmayer JM, Schoenfeld S, O'Grady R, Carney JK (1998) Methicillin-resistant Staphylococcus aureus in a high school wrestling team and the surrounding community. Arch Intern Med 158: 895-899. [Crossref]

15. Sosin DM, Gunn RA, Ford WL, Skaggs JW (1989) An outbreak of furunculosis among high school athletes. Am J Sports Med 17: 828-832. [Crossref] 
16. Brenner IK, Shek PN, Shephard RJ (1994) Infection in athletes. Sports Med 17: 86107. [Crossref]

17. Bibel DJ, Miller SJ, Brown BE, Pandey BB, Elias PM, et al. (1989) Antimicrobial activity of stratum corneum lipids from normal and essential fatty acid-deficient mice. J Invest Dermatol 92: 632-638. [Crossref]

18. Yamada Y, Fukunaga M, Hayashi K, Umehara Y, Nakao T (1991) Bacterial contamination of towels in the work of food service management. Suzuka Junior College 11: 121-127.

19. Anthony S, Jennifer S, Danielle MS (2010) Microbiology; a clinical approach. (1stedn), Garland Science, Taylor \& Francis Group, LLC, New York.

20. Yamaguchi J (2007) Rising with chlorhexidinegluconate shows comparable antibacterial effect to blushing on the preoperative skin treatment for THA. Hip Joint 33 : 19-21.

Copyright: (C2015 Eda N. This is an open-access article distributed under the terms of the Creative Commons Attribution License, which permits unrestricted use, distribution, and reproduction in any medium, provided the original author and source are credited. 